\title{
The odyssey of osteoclast precursors
}

$\mathrm{H}$ ow are bone-resorbing cells recruited to bone? Previously, Naoyuki Takahashi and colleagues identified dedicated precursor cells, which they termed "quiescent osteoclast precursors” (QOPs). Now, the group report in the Journal of Bone and Mineral Research that these cells, poised to become osteoclasts, circulate in the blood until they home to bone and differentiate. The findings, from studies in mice, increase understanding of the physiological control of bone turnover.

The differentiation of osteoclasts from cells in the monocyte/macrophage lineage is closely regulated by the expression of two key cytokines-colony stimulating factor 1 (CSF1) and receptor activator of NFkB ligand (RANKL) - that are produced by osteoblasts, responsible for bone formation. In 2009, Takahashi and colleagues found that, rather than originating from cells of a broad monocyte/macrophage lineage as previously thought, osteoclasts can differentiate from a group of specific, cell-cycle-arrested myeloid cells -QOPs. Found at the bone surface, QOPs differentiated into osteoclasts in response to CSF1 and RANKL without cell-cycle progression. In their new study, the group focused on the characteristics of QOPs from peripheral blood and bone marrow.

Florescence-activated cell sorting was used to isolate RANK and CSF1 receptor (CSF1R) double-positive osteoclast precursors from bone marrow. Three distinct cell populations were identified: RANK $^{\text {high }}{ }^{2}$ F $1 R^{\text {low }}$, RANK ${ }^{\text {high }} C S F 1 R^{\text {high }}$ and RANK ${ }^{\text {low }}$ CSF1R ${ }^{\text {high }}$. When cultured with RANKL and CSF1 for 5 days, these cells differentiated into tartrate-resistant acid phosphatase (TRAP)-positive osteoclasts. However, when this culturing was repeated with RANKL and CSF1 supplemented with hydroxyurea (an inhibitor of DNA replication that inhibits differentiation of osteoclasts from bone marrow macrophages), osteoclastogenesis was almost completely inhibited in

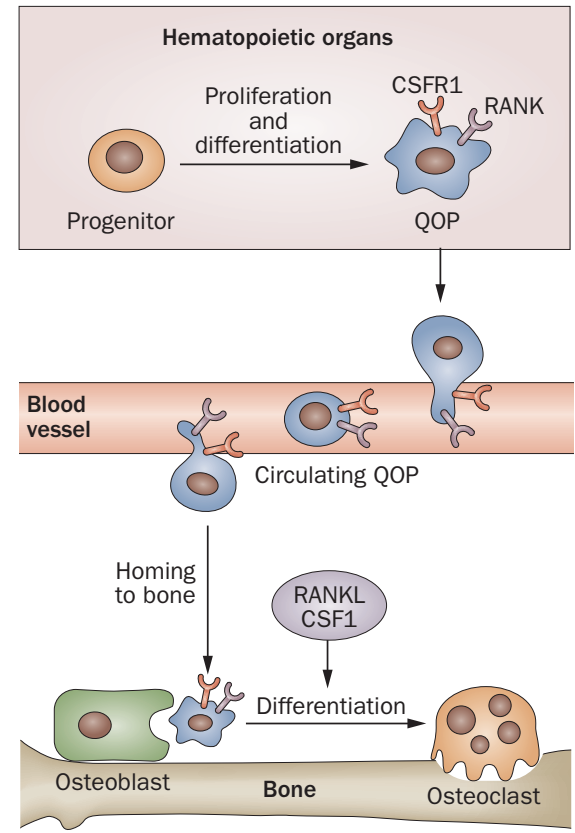

RANK ${ }^{\text {low }} C S F 1 R^{\text {high }}$ cells, significantly decreased in RANK ${ }^{\text {high }} C S F 1 R^{\text {high }}$ cells, and unchanged in RANK ${ }^{\text {high }} \mathrm{CSF} 1 \mathrm{R}^{\text {low }}$ cells. These findings suggested that the RANK ${ }^{\text {high }} C S F 1 R^{\text {low }}$ population contained mostly QOPs, able to differentiate without cell-cycle progression. Macrophage and monocyte lineage expression markers were expressed at low levels in these cells. Whereas RANK ${ }^{\text {low }}$ CSF1 $\mathrm{R}^{\text {high }}$ cells were able to differentiate into dendritic cells when treated with CSF2 and lipopolysaccharide, $\mathrm{RANK}^{\text {high }} \mathrm{CSF} 1 \mathrm{R}^{\text {low }}$ were not, confirming the lineage-determined nature of QOPs as previously described.

Next, collagen disks containing bone morphogenetic protein 2 (BMP-2) were implanted into dorsal muscle pouches in mice; ectopic bone formed after 2 weeks and TRAP-positive osteoclasts were detected on its surface. The mice were administered bromodeoxyuridine (BrdU) in their drinking water throughout the experiment: most of the nuclei of the osteoclasts were negative for BrdU, indicating that the cells differentiated from the cell-cycle arrested QOPs. By comparison, most of the osteocytes present in the ectopic bone were positive for BrdU, indicating that osteoblasts had formed from proliferative precursors.

BrdU was then used to determine the lifespan of $\mathrm{RANK}^{+}$cells compared with cells positive for F4/80, a marker of mature macrophages in mice. After 2 weeks of BrdU administration, $>80 \%$ of nuclei in F4/80+ cells were BrdU-positive, compared with $\sim 25 \%$ of RANK $^{+}$cells. A 4-week period in which 2 weeks of BrdU administration were followed by a 2 -week washout period resulted in roughly $10 \%$ of $\mathrm{RANK}^{+}$cells maintaining BrdU staining, whereas almost all of the F4/80 cells lost BrdU positivity. The researchers calculated the half-lives of F4/80 ${ }^{+}$and $\mathrm{RANK}^{+}$cells to be approximately 3 and 11 days, respectively, indicating a slow rate of proliferation and long lifespan of QOPs.

$\mathrm{RANKL}^{-1-}$ mice, which lack osteoclasts but possess QOPs, were then implanted with BMP-2-containing collagen disks or control disks that lacked BMP-2. After 2 weeks, the distribution of $\mathrm{F} 4 / 80^{+}$cells in ectopic bone did not differ between BMP-2 and control disks, whereas $\mathrm{RANK}^{+}$cells were far more numerous in the BMP-2 disks compared with control disks. When the mice were injected with RANKL, osteoclasts formed in the BMP-2 disks only. Thus, it seems that QOPs circulate in the blood and settle in the appropriate location for osteoclastogenesis. Nevertheless, the authors report, "the cellular mechanism of the egress of QOPs from hematopoietic organs into the bloodstream remains to be elucidated."

This study, in concert with previous observations, increases our understanding of bone turnover, and in future might help to combat aberrant bone deposition.

Nick Warde

Original article Muto, A. et al. Lineage-committed osteoclast precursors circulate in blood and settle down into bone. J. Bone Miner. Res. doi:10.1002/jbmr.490 